\title{
Factors Associated With Good Functional Outcome Despite Large Post- treatment Infarct Volume in the ESCAPE Randomized Controlled Trial
}

Aravind Ganesh, Bijoy K. Menon, Zarina Assis, Andrew M. Demchuk, Fahad S Al-Ajlan, Jeremy L. Rempel, Blaise W. Baxter, John Thornton, Nima Kashani, Mayank Goyal, Michael D. Hill, for the ESCAPE Trialists

Background and Aims: Improved outcomes after endovascular thrombectomy (EVT) are associated with smaller infarct volumes. However, some patients with large post-treatment infarct volume (PIV) fare well. We explored factors associated with large PIV and good outcome.

Methods: We identified ESCAPE patients (EVT/control) with PIV $\geq 75^{\text {th }}$ percentile, per 24-48-hour CT/MRI. We compared "discrepant cases"- 90-day modified Rankin Scale (mRS) $\leq 2$ despite high PIV - against "non-discrepant cases"(mRS $\geq 3$ ), examining imaging modality, ASPECTS locations, NIHSS, complications, demographics, and comorbidities using Fisher's exact for binary variables and Wilcoxon rank-sum for continuous variables. Multi-variable logistic regression was used to evaluate factors independently associated with large-PIV/good-outcome.

Results: Median PIV was 21mL(IQR 7-72mL, $\mathrm{n}=315) . \quad 80$ patients had PIV $\geq 72 \mathrm{~mL} ; 12(15.0 \%)$ were discrepant cases(mRS $\leq 2)$. Compared to non-discrepant cases, discrepant cases had lower baseline/24-hour/48-hour NIHSS (Figure 1).

A. One or more vascular risk factors

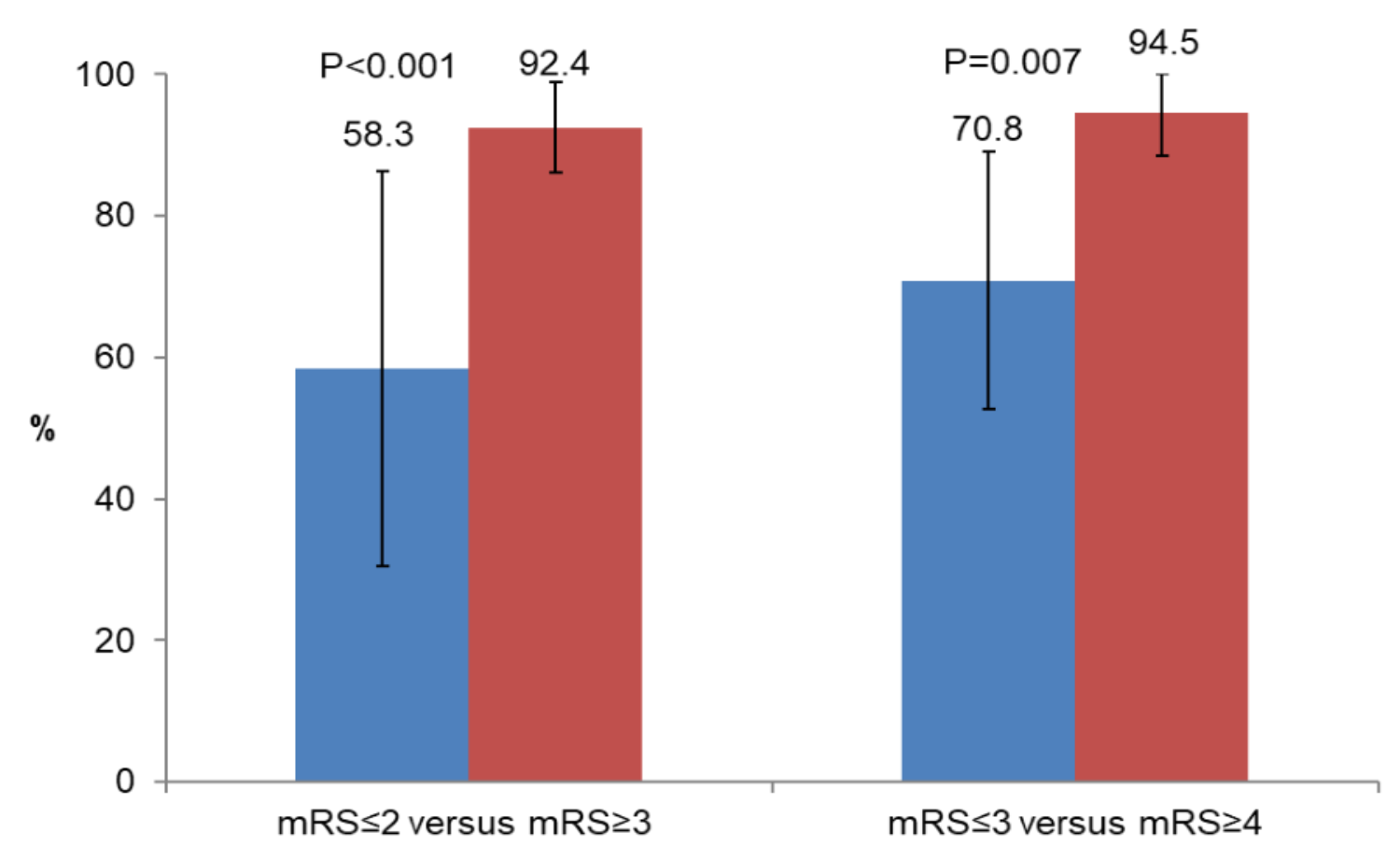

C. 48-hour NIHSS

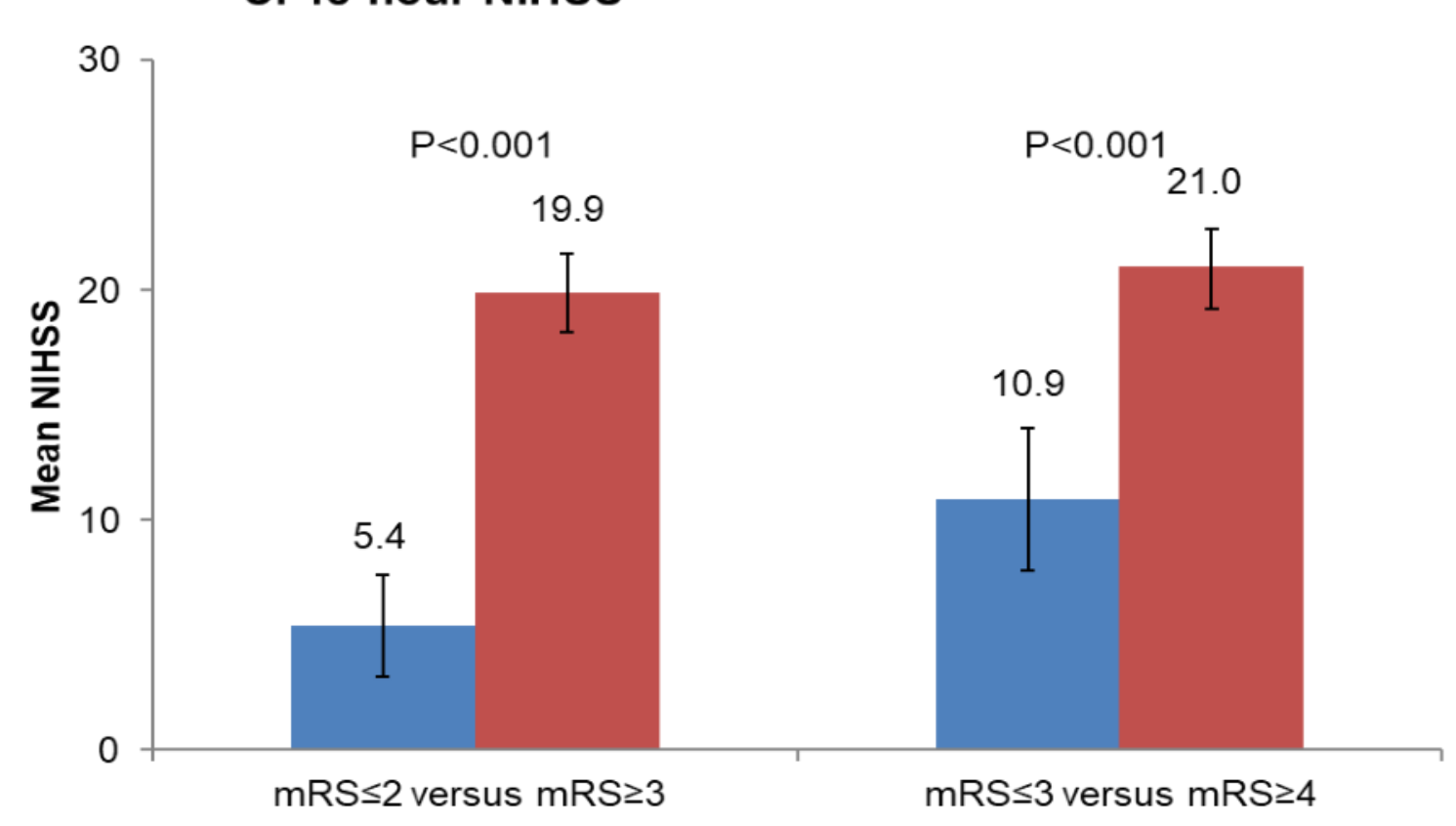

There were no differences in EVT use (33.3\% vs 40.3\%,p=0.76) or CT versus MRI volume ascertainment $(75.0 \%$ vs $87.9 \%, \mathrm{p}=0.36)$. On imaging, discrepant cases had higher ASPECTS at follow-up scanning (median 6 vs 2,p<0.0001), less frequent lentiform involvement, but no significant difference in laterality $(25.0 \%$ left vs $49.3 \%, p=0.21)$. They had lower systolic blood-pressure $\quad(134.7,124.2-145.1 \quad$ vs $152.7,145.1$ $160.2, p=0.03$ ) and less frequently had cardiovascular riskfactors, post-stroke complications, or malignant MCA infarctions (0 vs $25.4 \%, p=0.06$ ). On multi-variable regression, lentiform involvement $(p=0.02)$, 24-hour NIHSS $(p=0.006)$, and absence of cardiovascular risk-factors $(p=0.001)$ or malignant infarction(perfect) were independently associated with largePIV/good-outcome.

Conclusions: Good outcome despite large PIV is associated with absence of cardiovascular risk-factors or malignant infarction, lower NIHSS, and sparing of lentiform nucleus, the eloquence of which merits further study.

B. Involvement of the lentiform nucleus
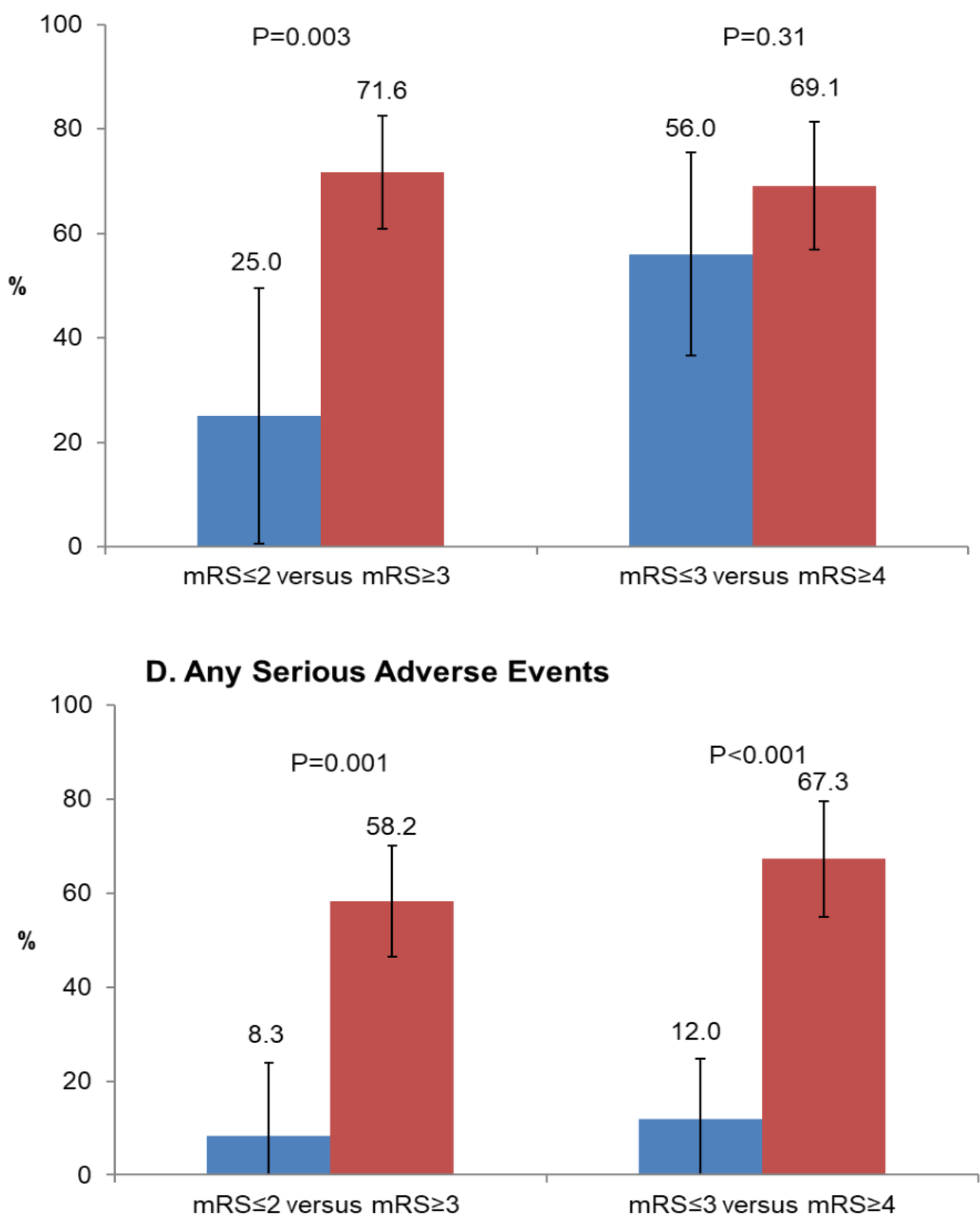

Figure 1 - Comparison of representative key variables that differed between patients with large PIV ( $\geq 72 \mathrm{~mL})$ and good outcome ("discrepant", blue, defined as either $m R S \leq 2$ or $m R S \leq 3$ ) and those with PIV $\geq 72 m L$ and poor outcome ("non-discrepant", red): (A) presence of vascular risk factors, (B) involvement of the lentiform nucleus, (C) 48-hour NIHSS, and (D) post-stroke SAEs. 